\title{
June 2015 Phoenix Critical Care Journal Club: Interventions in ARDS
}

Mortality has been declining in the adult respiratory distress syndrome (ARDS) (1). However, the cause of the decline in mortality is unclear. The only intervention shown to improve survival has been low tidal volume ventilation but the mortality was improving before this intervention was widely used (2). Nevertheless, it was suggested that we look at system performance regarding ARDS management from a critical appraisal standpoint. This journal club was hoped to help as a starting point in that regard. Four potential beneficial interventions were discussed: 1 . Conservative fluid management; 2 . Optimal PEEP as determined by esophageal pressure; 3 . Prone positioning; and 4. Mechanical ventilation driving pressure.

National Heart, Lung, and Blood Institute Acute Respiratory Distress Syndrome (ARDS) Clinical Trials Network, Wiedemann HP, Wheeler AP, Bernard GR, Thompson BT, Hayden D, deBoisblanc B, Connors AF Jr, Hite RD, Harabin AL. Comparison of two fluid-management strategies in acute lung injury. $N$ Engl $J$ Med. 2006;354(24):2564-75. [CrossRef] [PubMed]

Whether ARDS patients should be "dry" by diuresis and/or fluid restriction has been debated for at least 40 years. The ARDS network attempted to answer the question with a randomized study, comparing a conservative and a liberal strategy of fluid management. The primary end point was death at 60 days. Secondary end points included the number of ventilator-free days and organ-failure-free days and measures of lung physiology. During the study, the cumulative seven-day fluid balance was essentially zero in the conservative treatment arm and $7 \mathrm{~L}$ positive with liberal fluid management. The rate of death at 60 days was 25.5 percent in the conservativestrategy group and 28.4 percent in the liberal-strategy group $(P=0.30)$. Measures of lung physiology improved in the conservative-strategy group and the number of ventilator-free days $(14.6 \pm 0.5$ vs. $12.1 \pm 0.5, \mathrm{P}<0.001)$ and days not spent in the intensive care unit $(13.4 \pm 0.4$ vs. $11.2 \pm 0.4, P<0.001)$ also improved. The internal validity and generalizability of this study were both high. Some felt that these results support the use of a conservative strategy of fluid management in patients with acute lung injury while others felt that the benefit was small and pointed out that mortality was unaltered. There does not appear to be any benefit of fluid-overloading patients - and if avoiding $7 \mathrm{~L}$ overload gets patients extubated 2 days earlier, it seems worth pursuing.

Talmor D, Sarge T, Malhotra A, et al. Mechanical ventilation guided by esophageal pressure in acute lung injury. N Engl J Med. 2008;359(20):2095-104. [CrossRef] [PubMed]

Survival of patients with acute lung injury or the acute respiratory distress syndrome (ARDS) has been improved by ventilation with small tidal volumes and the use of positive end-expiratory pressure (PEEP); however, the optimal level of PEEP has been difficult to determine. In a pilot study, the authors estimated transpulmonary pressure with the use of esophageal balloon catheters. ARDS patients were randomized to undergo mechanical ventilation with PEEP adjusted according to measurements of 
esophageal pressure (the esophageal-pressure-guided group) or according to the ARDS Network standard-of-care recommendations (the control group). The primary end point was improvement in oxygenation. The secondary end points included respiratorysystem compliance and patient outcomes. This was a small RCT that only showed improvement in surrogate (not clinical) outcomes. Oxygenation and respiratory-system compliance improved $(P=0.01)$, and surprisingly for this small group, the 28-day mortality approached statistical significance $(P=0.055)$. It was pointed out that the use of esophageal balloons may be difficult to implement and maintain in the ICU. Studies are currently ongoing examining outcomes with this strategy and most felt that the results should be awaited before esophageal pressure monitoring has widespread implementation.

\section{Guérin C, Reignier J, Richard JC, et al. Prone positioning in severe acute respiratory distress syndrome. N Engl J Med. 2013;368(23):2159-68. [CrossRef] [PubMed]}

Previous trials involving patients with the acute respiratory distress syndrome (ARDS) have failed to show a beneficial effect of prone positioning during mechanical ventilatory support on outcomes. Yet, despite this lack of evidence, some have continued to use proning with the belief that studies would eventually show a benefit. For those intensivists, this study provides welcome positive evidence for proning. In this multicenter, prospective, randomized, controlled trial, 466 patients with severe ARDS were randomly assigned to undergo prone-positioning sessions of at least 16 hours or to be left in the supine position. The 28 -day mortality was $16.0 \%$ in the prone group and $32.8 \%$ in the supine group $(\mathrm{P}<0.001)$. The incidence of complications did not differ significantly between the groups. This RCT had strong internal validity and generalizability. The NNT to save a life is six. We noted that many previous interventions in critical care that were supported by only a single well-designed RCT were later found to be ineffective or even harmful (for instance: tight glycemic control, early goal-directed therapy of sepsis, and drotrecogin-alpha for sepsis). We therefore view implementation of proning with some skepticism, but felt it may be under-utilized. It's best application seems to be early in the course of severe ARDS. We all worried about the practicality of proning our patients, especially those with morbid obesity, but felt that if we practiced proning more frequently, we would gain practical experience.

\section{Amato MB, Meade MO, Slutsky AS, et al. Driving pressure and survival in the acute respiratory distress syndrome. N Engl J Med. 2015;372(8):747-55. [CrossRef] [PubMed]}

The newest concept to be discussed was driving pressure during mechanical ventilation. Mechanical-ventilation strategies that use lower end-inspiratory (plateau) airway pressures, lower tidal volumes (VT), and higher positive end-expiratory pressures (PEEPs) can improve survival in patients with the acute respiratory distress syndrome (ARDS), but the relative importance of each of these components is uncertain. Because respiratory-system compliance (CRS) is strongly related to the volume of aerated remaining functional lung during disease (termed functional lung 
size), the authors hypothesized that driving pressure ( $\triangle \mathrm{P}=\mathrm{VT} / \mathrm{CRS})$, in which $\mathrm{VT}$ is intrinsically normalized to functional lung size (instead of predicted lung size in healthy persons), would be an index more strongly associated with survival than VT or PEEP in patients who are not actively breathing. The authors examined the records of 3562 patients with ARDS enrolled in nine previously reported randomized trials and found that a 1-SD increment in $\triangle \mathrm{P}$ (approximately $7 \mathrm{~cm}$ of water) was associated with increased mortality (relative risk, 1.41; 95\% confidence interval [Cl], 1.31 to 1.51; $\mathrm{P}<0.001$ ), even in patients receiving "protective" plateau pressures and VT (relative risk, $1.36 ; 95 \% \mathrm{Cl}, 1.17$ to $1.58 ; \mathrm{P}<0.001$ ). This study has strong internal validity, but was only hypothesis-generating in design. The concept of using driving pressure makes physiological sense, yet it is unclear if altering $\Delta \mathrm{P}$ improves survival or if this is merely an independent marker of survival. This study invites prospective trials.

Richard A. Robbins, MD

Robert A. Raschke, MD

\section{References}

1. Abel SJ, Finney SJ, Brett SJ, Keogh BF, Morgan CJ, Evans TW. Reduced mortality in association with the acute respiratory distress syndrome (ARDS). Thorax. 1998;53(4):292-4. [CrossRef] [PubMed]

2. Kallet RH, Jasmer RM, Pittet JF, Tang JF, Campbell AR, Dicker R, Hemphill C, Luce $\mathrm{JM}$. Clinical implementation of the ARDS network protocol is associated with reduced hospital mortality compared with historical controls. Crit Care Med. 2005;33(5):925-9. [CrossRef] [PubMed] 